\title{
Emerging strategies and applications of pharmacogenomics
}

\author{
Patrice M. Milos* and Albert B. Seymour \\ Discovery Pharmacogenomics, Pfizer Global Research and Development, Eastern Point Road, Groton, CT 06340, USA \\ *Correspondence to: Tel: +1 860441 4342; Fax: +1 860686 1164; E-mail: Patrice_m_milos@groton.pfizer.com
}

Date received (in revised form): 6th October 2004

\begin{abstract}
The rapid pace of genomic science advancements, including the completion of the human genome sequence, the extensive cataloguing of genetic variation and the acceleration of technologies to assess such variation, combined with clinical programmes with rich phenotypic data, serve as the foundation for the design and execution of pharmacogenomic studies which have an impact on the pharmaceutical pipeline from early discovery through to the marketplace. The authors discuss the required infrastructure to support pharmacogenomic studies and provide insight into the strategies and practical application to influence decision making in the pharmaceutical setting. Further, the influence of pharmacogenomics is currently affecting patient care in the oncology area and is highlighted as evident impact in the marketplace.
\end{abstract}

Keywords: pharmacogenetics, pharmacogenomics, single nucleotide polymorphisms (SNPs), genotype-phenotype association

\section{Introduction}

Pharmaceutical engagement of the pharmacogenomics discipline gained significant momentum in the mid-1990s as colleagues within the industry recognised the potential opportunity for the emerging field to have an impact on various phases of the drug discovery and development processes. While the optimism and excitement for this emerging discipline was high, the scientific and technological hurdles required to deliver pharmacogenomics seemed significant. At the time, the demonstration of pharmacogenomic impact as embodied by the drug metabolism field was evident, yet many hurdles remained to enable the delivery of comprehensive pharmacogenomic studies to explore disease state and drug response relationships. These hurdles included a complete draft sequence of the human genome, not expected until 2005. From this sequence would then emerge the catalogue of human genome variation, and this was not expected until several years after the completion of the human genome sequence. Speculation at the time suggested that more than 300,000 evenly spaced, single nucleotide polymorphisms (SNPs) would be needed to perform comprehensive pharmacogenomic studies - a scale unimaginable at the time. ${ }^{1,2}$ How could it be possible to catalogue the SNPs needed to perform whole-genome association studies and conduct the genotyping - the building blocks speculated to drive the scientific revolution needed to fully deliver pharmacogenomics?

Over the past ten years, significant technological progress to enable pharmacogenomics has been achieved.
The International Human Genome project and Celera raced to publish the working draft genome sequence in $2001^{3,4}$ four years ahead of schedule, and the SNP Consortium, formed in 1999 through partnership between the academic and industrial community, developed the first comprehensive SNP map, published in November 2000, ahead of schedule, below cost and exceeding the initial goal of identifing 300,000 SNP markers of which 150,000 would be mapped by some $1.12 \mathrm{M}$, delivering $1.42 \mathrm{M}$ mapped SNPs for the scientific community at large to utilise for scientific research. ${ }^{5,6}$ This has now evolved into the International HapMap project, which will further refine the SNPs required for comprehensive association analysis by identifying critical patterns of SNPs that cosegregate from generation to generation in the form of haplotype tags and may provide the framework from which human whole-genome association studies are designed. ${ }^{7}$

In parallel with the rapid advancements in the identification and mapping of SNPs across the human genome, technological discoveries and enhancements to high-throughput genotyping have also made significant strides. Over the course of the last five years, leading biotech firms have driven genotyping throughput to scales in the order of millions per day while significantly driving down costs. These technologies are now available to human genetics scientists to support large multi-gene association studies and the early application of human whole-genome association studies.

So, while in the mid-1990s many people heralded the revolution of 'personalised medicine' and 'the right drug for 
the right patient', the realists understood that, like any revolution, scientific research and technological advances would be required to shape the potential importance of this field to modern medicine. It is now understood that any two individuals differ from each other at the DNA sequence level by a mere 0.1 per cent. Yet it is this 0.1 per cent difference which makes each person unique and holds the key to differences in disease susceptibility and progression, as well as our response to therapeutic intervention. ${ }^{8}$ As of now, the foundation has been laid for the broadening application of pharmacogenomics; clear examples exist in medical practice, and scientific experiments can now commence at a pace and level which would have seemed unimaginable back in the mid-1990s. Furthermore, the face of the pharmaceutical industry has changed dramatically; with ever-increasing development costs and decreasing numbers of new drug approvals, the challenges facing the industry have never been more significant. This paper aims to highlight the infrastructure needed to integrate pharmacogenomics into the pharmaceutical business, as well as strategies for application, and discusses the potential applications and opportunities that this field offers to influence medical care.

\section{Infrastructure for delivering pharmacogenomics into the pharmaceutical setting}

The discipline of pharmacogenomics is currently being applied throughout the drug discovery and development process, as depicted in Figure 1. Highlights include: applying human genetics to ensure that the best therapeutic targets are prioritised for discovery investment; the comprehensive evaluation of the target gene sequence in multiple subjects to determine the genetic heterogeneity present in different ethnic populations; the use of genetics to select particular subpopulations and in early proof of concept studies; and, finally, the potential to predict efficacy and safety of new medicines more precisely. Prior to the effective integration and application of pharmacogenomics, however, significant investment and infrastructure was required to integrate pharmacogenomics into these processes. These areas are highlighted in Figure 2 and discussed in more detail below.

\section{Appropriate informed consent}

The ability to collect DNA, as well as other types of genomic samples, from study subjects is directly dependent on patient informed consent as to the risks and benefits associated with the sample collection. A separate informed consent, which states the purpose and the use of the samples, assurance of patient confidentiality through anonymisation or de-identification of unique patient identifiers and clinical data, as well as the optional participation in this donation, has been an integral part of patient acceptance in the clinical trial setting. Within current Pfizer clinical programmes it is not unusual for patient consent for genomic sample collection to exceed 80 per cent in clinical studies taking place in countries around the world.

\section{Collection of phenotypic data}

The ability to execute any genetic study requires accurate and comprehensive phenotypes, which at best are quantitative and reliable, to enable robust genetic analyses. Clinical trials serve as an excellent starting point for genetic research aimed at better understanding the underlying disease state and providing clinical results including outcome measures and drug response, both of which can drive pharmacogenomic investigations. Clinical trials are not genetic studies by design, however, and critical review of the completed study will often require careful epidemiological evaluation to ensure the appropriate study design. This often involves ensuring that those subjects donating DNA samples are reflective of the entire study population and are not biased in any way. If one is interrogating drug response phenotypes, care must be taken to examine the demographics of the responder/non-responder subgroups. Similarly, when safety events are investigated, subjects experiencing the adverse event must be closely matched to those subjects receiving the drug and exhibiting no such adverse event.

When considering the use of clinical trial samples for disease gene interrogations, the ascertainment of appropriately matched control populations to support such genetic studies is equally important. Often, clinical methodology studies to ascertain control populations matched on demographics as well as on certain clinical phenotypes present in the case population are required. In the authors' studies of osteoarthritis, critical care was taken to execute a study which identified control individuals who were radiographically free of joint space narrowing, to ensure accurate control phenotype status. ${ }^{9}$ These collections then allow the design and execution of case-control association studies in support of both target validation and genetic biomarker discovery to support clinical development programmes targeting the specific disease.

\section{Technology for delivery of genetic analysis} The rapid pace of technology advancements enables the interrogation of the entire genome at the level of DNA, RNA or protein. Technology has moved rapidly from methods of restriction fragment polymorphism (RFLP) scoring of genetic variation to examining individual gene markers associated with various phenotypes, ${ }^{10}$ to SNP scoring to examine multiple markers in multiple candidate genes, ${ }^{11}$ to now having the ability to interrogate thousands of SNP markers using methods typified by Sequenom mass spec, ${ }^{12}$ Illumina bead-based methods, ${ }^{13}$ the Affymetrix high-density SNP chips $^{14}$ and Perlegen high-density wafers. ${ }^{15}$ It is now possible to examine hundreds of millions of SNPs within several weeks, as the 


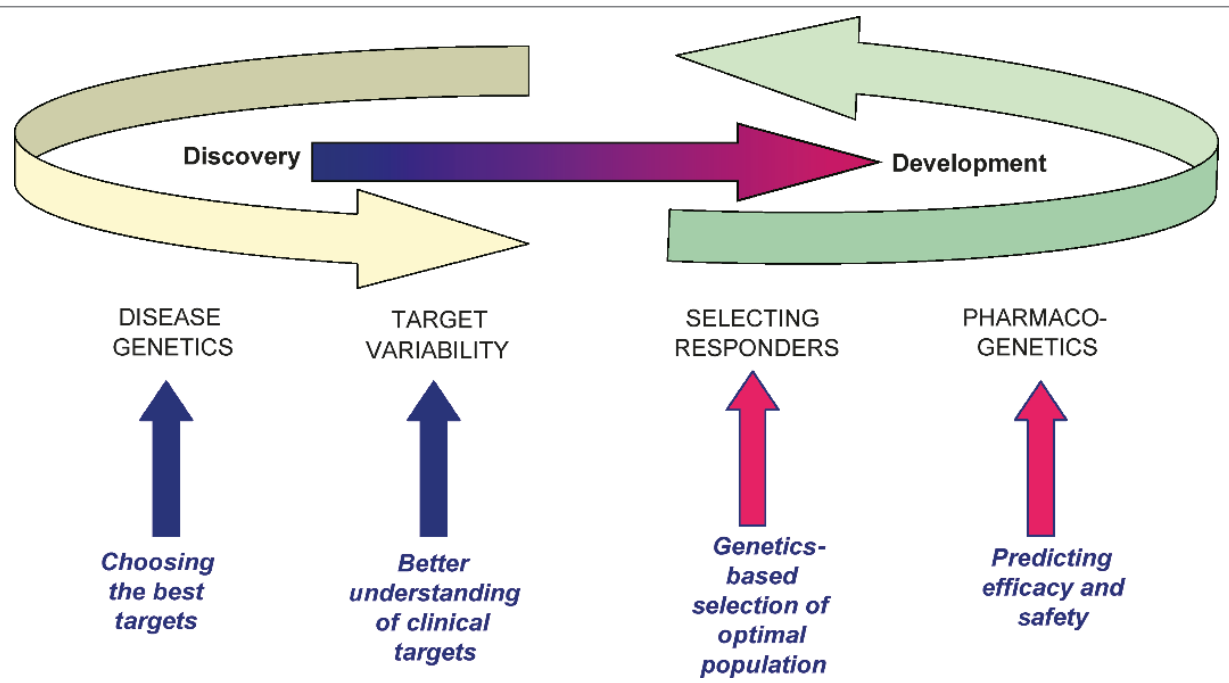

Figure I. Applying pharmacogenomics to deliver new medicines. The ability to integrate pharmacogenomics into the drug discovery and development process is a continuum with applications at each step of the process. This diagram highlights the various phases of pharmaceutical application which are highlighted in this paper.

authors are doing through their collaboration with Perlegen Sciences to study metabolic syndrome and depression phenotypes. It is anticipated that whole-genome sequencing in a timely and cost-effective manner may represent the next technological floodgate. ${ }^{16}$

\section{Information technology}

One of the single most important challenges facing the field of pharmacogenomics rests in the hands of the information technology experts. The vast amount of clinical data, combined with genomic data, requires a well designed information architecture to enable hypothesis generation at one end of the portfolio and validated systems to enable the use of the information in regulatory submissions at the other end. For example, one must be able to track clinical genomic samples from the patient from sample processing through to the sample repository, ensuring that there is integrated information regarding informed consent and potential restrictions on

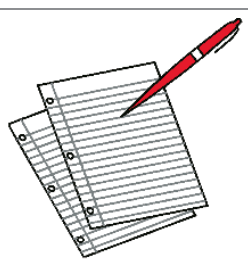

Informed consent

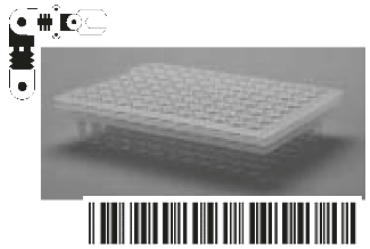

Genotyping platforms

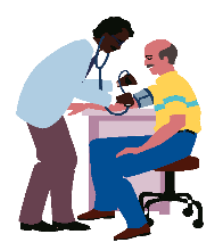

Phenotypic data collection

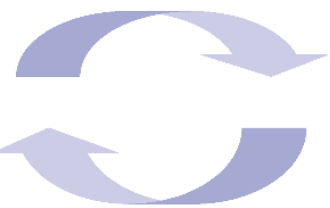

Application to

clinical

development

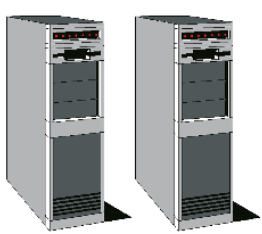

Information systems

Figure 2. Infrastructure requirements for pharmacogenomics. Much investment in various tools and technologies are required to ensure the seamless integration of pharmacogenomics into the pharmaceutical setting to deliver value and impact throughout the pipeline. 
sample use, and that clinical data associated with the matched genomic sample is entered into a database. Moreover, often, a final step of anonymisation or de-identification must occur prior to initiation of a pharmacogenomic study. At this stage, the challenge lies in the ability to effectively query the clinical data in order to develop specific hypotheses that can be addressed by pharmacogenomic investigations, followed by data systems that will then house all of the newly generated genomic data, often derived from very different types of analysis platforms.

The ability to effectively integrate these data with clinical data and to mine such datasets remains elusive, and will require a comprehensive information technology solution to maximise the utility of research studies. This sophisticated knowledge base becomes a primary limitation in many cases, as the ability to integrate and effectively mine disparate data sources remains challenging and time intensive; however, this will have to be addressed in sophisticated systems to enable maximum return on investments in pharmacogenomics. In addition, when the importance of patient confidentiality in the future application of pharmacogenomics in medical care is considered, significant gaps exist. New business models have emerged that aim to focus on the integration and confidentiality of data sources. These include models developed by First Genetic Trust ${ }^{17}$ and the Clinical Genomics business unit of IBM.

\section{Carrying out pharmacogenomics studies to deliver value}

Within the industry setting, the ability to drive pharmacogenomics into the fabric of the business is directly dependent on the potential to have an impact on business decisions. This therefore requires close partnership with colleagues in discovery, development and commercial marketing to work together to shape the scientific direction of research programmes. Given the challenges of the pharmaceutical industry, including the high attrition which occurs at every stage throughout the pipeline, combined with the long development time lines, excellent science alone is not sufficient. Pharmacogenomics has to drive impact and influence decisions aimed at increasing productivity within drug discovery and clinical development. A discussion focused on achieving such value follows.

\section{Choosing the best target: Applying human genetics to add human relevance}

There exist significant unmet medical needs of patients, and it is now recognised that common diseases effecting morbidity and mortality have a significant genetic component. Consider the diseases of diabetes, obesity and osteoarthritis, to name but a few. The degree of genetic contribution has been estimated for these diseases based on twin studies and sibling risk analysis. ${ }^{18-23}$ Similarly, rare gene mutations have provided insight into complex biological processes involved in more common disease phenotypes. For example, the importance of cholesteryl ester transfer protein (CETP) in influencing an individual's high-density lipoprotein (HDL) levels was demonstrated when individuals identified as possessing extreme levels of HDL were shown to harbour a rare mutation in their CETP gene and to lack CETP activity. $^{24,25}$ In another example, subjects harbouring inactivating mutations within the Janus kinase 3 gene (JAK3) express severe combined immunodeficiency syndrome (SCID), providing mechanistic evidence that JAK3 inhibition was likely to effect immune suppression and potentially serve as a therapy for transplant recipients. ${ }^{26}$ Most importantly, the insight gained from the human genetics findings described above has led to new investigational drugs for CETP inhibition and JAK3 inhibition, which, upon entry into the clinic, demonstrate efficacy consistent with the human genetics data. $^{27,28}$

While not traditionally defined as pharmacogenomics, human genetic association studies may provide an important avenue for establishing relationships between human genes and disease state and thus influence the appropriate selection of therapeutic targets. Important new discoveries of genes associated with complex disease appear to occur on a regular basis now, as evidenced by disease associations of neuregulin and schizophrenia; ${ }^{29}$ PDE4D and stroke; ${ }^{30}$ and 5-lipoxgenase and atherosclerosis. ${ }^{31}$ These studies have been strengthened by additional scientific data supporting functional relationships between the genetic association and the disease.

Pharmaceutical companies, as well as many academic institutions, have been collecting DNA samples with appropriate informed consent to investigate the relationship between genetic variation and disease phenotypes to better classify disease. Through the collection of clinical phenotypes with linked DNA samples, the execution of clinical trials provides an opportunity to investigate the underlying genetic variation that exists in patients. This is typified in a study in which DNA from patients who participated in a lipidlowering trial demonstrated a strong association between the phenotype for HDL levels and a novel lipase gene family member, endothelial lipase $(L I P G) .{ }^{32}$ While association studies have had mixed reviews in the scientific community, often due to conflicting study results, careful attention to study design can provide the first opportunity to add human data to known and novel genes. Study design issues include: careful selection of appropriate study subjects based on rigorous phenotypic criteria; utility of extreme phenotypes; comprehensive SNP selection which takes into account linkage disequilibrium (LD) in the gene under study to ensure a density of markers which capture the LD pattern of the gene; statistical methods allowing one to assess how likely any result may be due to 
chance alone; and, finally, planned replication to provide added validation of any gene association. These are critical to the successful characterisation of disease risk alleles.

While candidate gene studies have become commonplace in the literature, these studies may often involve a biological hypothesis for candidate gene selection. The ability to interrogate the genome based solely on phenotypic criteria with no a priori bias on gene selection has been dramatically enhanced by technologies which now interrogate SNPs broadly across the genome. These platforms have now replaced estimates of 300,000 SNPs evenly spaced across the genome, by utilising some 200,000-300,000 haplotype-defining SNPs across the genome. For the first time, genotyping technologies — such as those developed by Perlegen Sciences have provided the ability to genotype hundreds of thousands of markers in an individual using high-density oligonucleotide arrays coupled with either highly multiplexed polymerase chain reactions or restriction enzyme-based genomic reduction. Even with these new technologies and the availability of millions of mapped SNPs, however, the exact number of haplotype-defining SNPs that will be required to detect genetic associations with common polymorphisms that are not typed is still uncertain. The appropriate LD measure to select these tagging SNPs for association studies is $r^{2} .33$ Recent reports evaluating common polymorphisms across candidate gene regions suggest that, in order to detect $>80$ per cent of all haplotypes in a given region using haplotype-tagging SNPs, it is necessary to reach an $r^{2}$ of $>0.8 .{ }^{34}$ As the HapMap project progresses forward with defining the linkage LD patterns in the human genome, geneticists will have the tools to evaluate the extent of LD within a given region and within a given ethnicity to select the optimal SNPs to genotype when designing a study. These data will provide valuable information around SNP selection, irrespective of whether the study design is for a whole-genome association or candidate gene-based studies. Elucidating the genetic contributors to common complex diseases, such as cardiovascular disease and psychiatric disease, will be more efficient through a whole-genome approach, as it does not depend on the biased selection of candidate genes. This has been exemplified using family-based linkage studies in stroke, ${ }^{30}$ schizophrenia ${ }^{29}$ and Crohn's disease, ${ }^{35}$ where these genes would not have been suspected a priori and thus not even investigated using a candidate gene approach. Whether the study design focuses on disease, drug response or safety, the primary driver for any successful study is the accurate clinical phenotyping of the study population. The present authors believe that this new information on SNP density and LD coverage of the human genome will allow the visualisation of a significant portion of the genomic regions associated with the phenotype of interest, as illustrated in Figure 3.

In an attempt to validate the haplotype tagging approach to identify genetic associations, as well as to evaluate the Perlegen Sciences array-based chip-based genotyping platform, 7,283 SNPs spanning 17.1 megabases (Mb) of DNA were genotyped to identify associations with HDL levels. ${ }^{36}$ SNPs associated with the $5^{\prime}$ haploblock of the CETP gene were identified as the most significant association in the

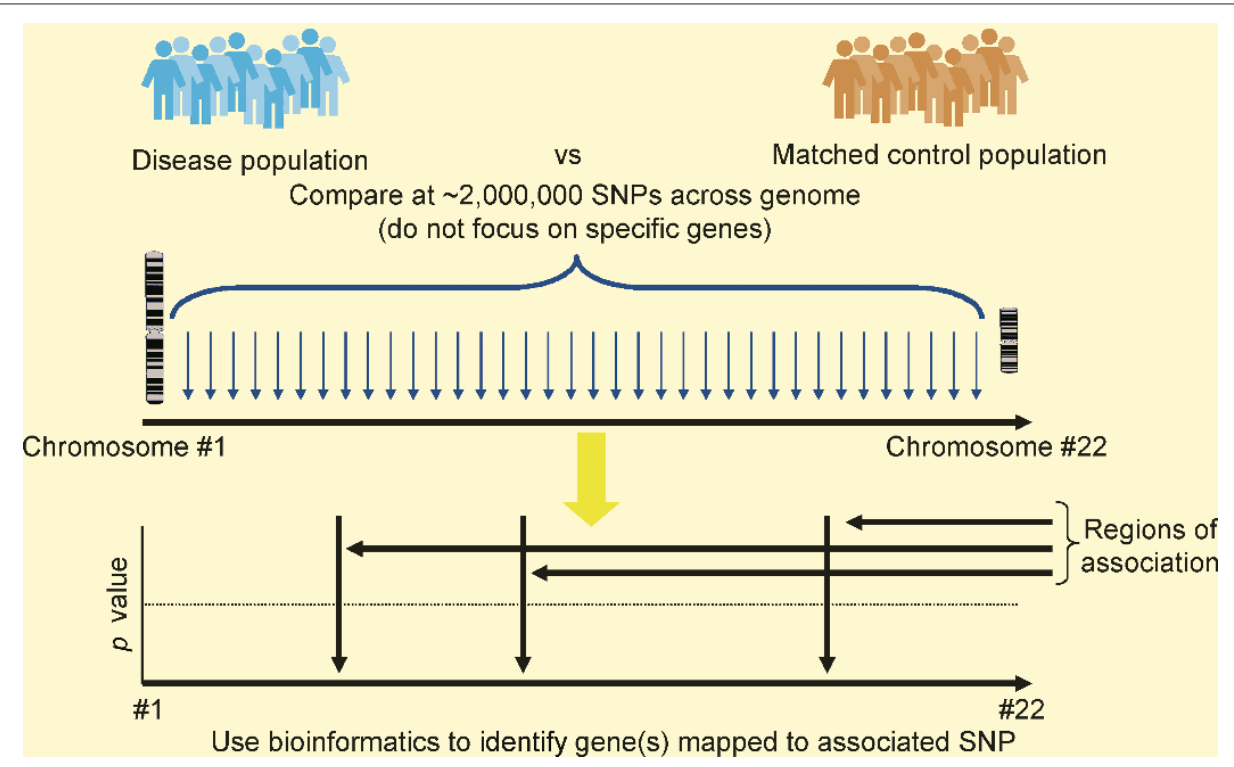

Figure 3. Exploiting whole-genome approaches. Candidate gene-based studies have been the focus of human genetic association studies for the past several years. As the knowledge of genetic variation has emerged and the genotyping technologies to investigate such variation have matured, our ability to carry out whole-genome association studies appears poised to address fundamental issues, such as the association with complex diseases and drug responses. 
dataset. ${ }^{36}$ Readouts from several genome-wide studies are expected within the next year and will provide further insight into the potential value of these studies to identify genes involved in complex disease phenotypes, including metabolic syndrome and drug response. The public HapMap project ${ }^{7}$ and companies like Perlegen, which recently announced its intention to provide its SNP markers into the public domain to further advance the foundation for such experiments, will further aid these whole-genome approaches for the scientific community.

\section{Drug discovery and development: Understanding target variability}

Early in the drug discovery phase, the assessment of comprehensive target variation achieved through gene resequencing in ethnically diverse DNA panels provides discovery scientists with information that is critical for screen design, functional assessment of variant alleles and animal model design. In partnership with companies like Genaissance Pharmaceuticals, the present authors will typically screen a panel of 92 DNA samples with representations from Caucasian, Asian, Hispanic and African-American DNA samples, which allow the detection of polymorphisms with a frequency greater than 1 per cent. ${ }^{37}$ For example, the CETP gene variation depicted in Figure 4 provides a comprehensive understanding of the variation within this gene locus. In addition, these comprehensive SNP data provide the basis for candidate gene-based studies to interrogate the relationship between putative therapeutic target genes and human disease phenotypes. $^{38}$ This knowledge of target variation can then be utilised for hypothesis testing in early clinical programmes, with particular emphasis on putative functional variants which may have been defined during discovery studies exploring functional activities. This is exemplified in the following discussion.

\section{Drug development: Traditional pharmacogenetics for clinical decision making}

Most, if not all, pharmaceutical companies have actively engaged in the area of pharmacogenomics with differing levels of investment, strategies and application. It is the authors' belief, however, that application of this discipline is still in its infancy. Yet there are some very clear demonstrations of impact already in place, with particular emphasis on the oncology area, which are described in more detail in the next section.
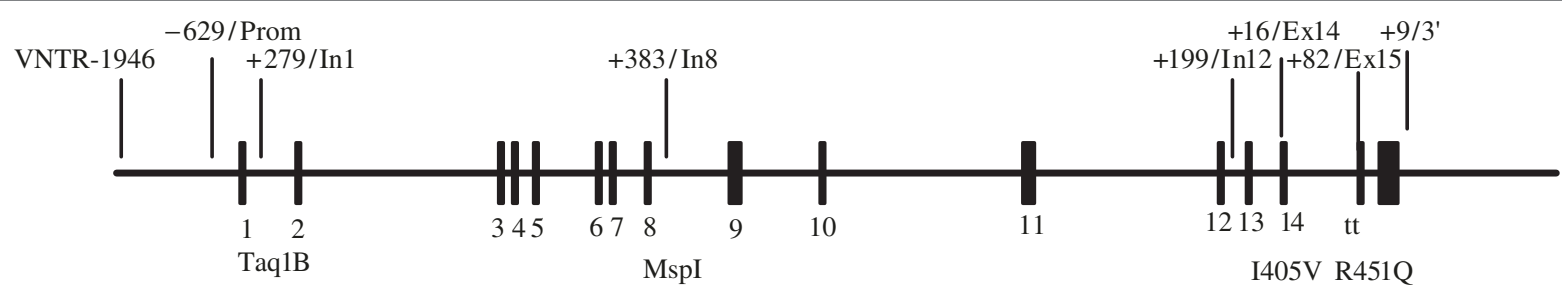

Minor allele frequencies

\begin{tabular}{|l|l|l|r|r|}
\hline Allele & dbSNP & Other name & \multicolumn{1}{l|}{ Caucasian } & \multicolumn{1}{c|}{ Af. Amer. } \\
\hline VNTR L/S & & -1946 & 0.56 & 0.67 \\
\hline C-629A/Prom & rs1800775 & & 0.09 & 0.02 \\
\hline G+279A/In1 & rs708272 & Taq1B & 0.49 & 0.55 \\
\hline A+383G/In8 & rs15723185 & Msp I & 0.18 & 0.24 \\
\hline 12 bpdel +199/In12 & & & 0.11 & ND \\
\hline C+408T/In12 & rs1800774 & & 0.28 & 0.45 \\
\hline A+16G/Ex14 & rs5882 & I405V & 0.18 & 0.24 \\
\hline G+82A/Ex15 & rs1800777 & R451Q & 0.07 & 0.2 \\
\hline G+9C/'3 & rs289742 & & 0.12 & 0.352 \\
\hline
\end{tabular}

Figure 4. Structure of the cholesteryl ester transfer protein (CETP) gene and polymorphic markers. The CETP gene is located on chromosome 16 and contains a number of polymorphisms which have been identified through dense resequencing. These polymorphisms occur at differing frequencies in various ethnic populations, as shown in the accompanying table. In this table, the single nucleotide polymorphism (SNP) marker is identified and the db SNP identifier along with other common names for the SNP and minor allele frequencies for Caucasians and African Americans (Af. Amer.). ${ }^{38}$ 
One can also look to the area of drug metabolism, the more classically defined area of pharmacogenetics, and find clear demonstrations of the utility of genetic knowledge of the cytochrome P450 gene family, as well as other genes involved in the pharmacokinetics of drug disposition. These genes have been extensively studied, and examples such as cytochrome P450 CYP2D6, 2C19 and 2C9 provide a clear demonstration of the predictive use of genotype in clinical development. For example, the use of genotyping to predict poor metaboliser status for individuals deficient in CYP2D6 enzyme is used routinely when evaluating CYP2D6 substrates. ${ }^{39,40}$ Many excellent reviews have been published, providing detailed summaries of the area of drug metabolism and its importance for clinical development. ${ }^{41-45}$

In the early stages of clinical development, CYP2D6 genotyping is routinely employed for prospective enrolment or retrospective analysis of subjects, to rapidly establish individual pharmacokinetic differences for those compounds suspected to be metabolised via the CYP2D6 pathway. ${ }^{40}$ Additionally, drug-drug interaction studies with experimental therapies and known CYP2D6 substrates, such as paroxetine, can benefit from the incorporation of CYP2D6 genotyping. ${ }^{46,47}$ CYP2D6 poor metabolisers may demonstrate altered paroxetine exposure, which would remain unexplained in the absence of CYP2D6 genotyping, and thus incorporation of the genotyping data removes the potential for uncertainty in those individuals who would be expected to demonstrate increased exposure due to the possession of two non-functional alleles of CYP2D6.

\section{Drug development: Transitioning to proof of concept}

The combined knowledge of genetic contributions to the underlying disease state and comprehensive data on target variation, provides new and valuable insight which can be applied in early clinical studies aimed at establishing proof of concept for novel therapeutic agents. Genetic biomarkers defining the risk of disease onset and/or progression can potentially be used to select optimal patient populations in early clinical development by targeting a genetically defined disease risk population relevant to the therapeutic intervention or the endpoint being studied. This is exemplified by recent data from deCODE Genetics, in which genetic variation within the 5-lipoxygenase activating protein (FLAP) gene locus was associated with an increased risk of myocardial infarction in the Icelandic population. ${ }^{48}$ Functional assessment of isolated neutrophils from individuals carrying the 'at-risk' four-SNP marker haplotype demonstrates increased production of leukotriene B4, a major leukotriene in this inflammatory pathway. This unique haplotype identifies a specific population in which to assess the potential therapeutic benefits of a FLAP inhibitor, and deCODE Genetics has initiated clinical studies to validate this approach, combining genetic enrichment with related biochemical measures of drug activity and inflammation to assess proof of concept. Should this study prove successful, the question will remain as to whether this mechanism will be beneficial in the genetically 'at risk' FLAP haplotype individuals alone, or whether other individuals will also benefit from therapeutic intervention directed at this specific mechanism. This is a bold new approach which has encouraged Merck to partner with deCODE Genetics, and will probably be watched carefully by other pharmaceutical companies.

The utility and importance of target variation data and knowledge about the functional relevance of this variation to execute pharmacogenomic studies in early development has also been applied in the development of novel therapies for HIV. Genetic studies first demonstrated that the CCR 5 receptor was associated with susceptibility to HIV infection and the progression to AIDS. ${ }^{49}$ The CCR 5 gene has been well characterised, with particular importance placed on the delta 32 allele, as individuals heterozygous for the allele exhibit significant reduction in receptor numbers on the cell surface while individuals homozygous for the allelle have no functional receptor present. Pharmaceutical companies have therefore rapidly moved CCR 5 receptor antagonists from discovery into the clinic. In early clinical studies of a CCR 5 antagonist, examination of the delta 32 genotype and receptor density as measured by fluorescence-activated cell sorting analysis, has shown that the CCR 5 delta 32 was not significantly associated with any safety and toleration endpoints tested, nor with baseline viral load. Preliminary investigation suggests no apparent relationship between CCR 5 delta 32, CCR 5 expression and receptor saturation in healthy volunteers. Future studies will examine the CCR 5 genotype and antiretroviral activity as the compound progresses through clinical development. ${ }^{50}$

\section{Utilisation in late-stage clinical development programmes and beyond}

It is often difficult to ascertain individual pharmaceutical experience in late-stage clinical development, as much experience to date has involved retrospective analysis of clinical programmes and has not moved into true prospective application. In addition, there still exists a significant rate of late-stage attrition. A recent publication from the US Food and Drug Administration (FDA) provided some insight into the integration of pharmacogenomics into clinical development programmes, citing some 70 investigational new drug applications involving pharmacogenomics; of these 80 per cent involved cytochrome P450 genotyping and were related to drug metabolism. ${ }^{51}$ Recent issuance of the draft guidance for Voluntary Genomic Data Submissions by the FDA will also promote 
additional regulatory dialogue and information sharing related to the application of pharmacogenomics.

Roses recently provided an excellent example with Tranilast, a Phase III programme which was unsuccessful for restenosis, in which pharmacogenomics data were generated in real time during the Phase III development programme, demonstrating that a small percentage of patients developing hyperbilirubinaemia, during drug treatment contained a promoter variant in the UDG-glucuronosyltransferase I gene (UGT1); this was not seen in placebo-treated subjects. ${ }^{52,53}$ This initial Tranilast work has now been extended to model SNP scoring at a density expected for whole-genome scans and to serve as a validation of whole-genome analysis for identifying genomic regions associated with safety events. Some 76 haplotype-defining SNPs, spanning a $2.7 \mathrm{Mb}$ region of DNA including the UGT1A1 gene, were examined in 1,054 patients who had been treated with Tranilast with no evident hyperbilirubinaemia, and 147 patients receiving therapy who did experience hyperbilirubinaemia. This analysis demonstrated an association in a 150 kilobase region that included the UGT1A1 gene locus, which upon further dense mapping confirmed the association with the UGT1A1 gene. ${ }^{54,55}$

Table 1 describes other examples of currently available drugs that have been examined for genetic associations with particular genetic pathways or gene markers. These references provide an early window onto the types of studies which are exploring the complex relationship between drug response and genetic heterogeneity in relatively small numbers of individuals receiving such therapies. These have relied on focused candidate gene-based investigations with limited ability to interrogate the entire genome; however, it is envisioned that several such genome-wide analyses will appear in the scientific community over the coming year or two.

\section{Utilisation of pharmacogenomics in oncology to have an impact on patient care}

Cancer is a genetic disease, both in terms of the germline (inherited genome) as well as the somatic genome, which is unique to the tumour itself. While mutations in genes such as $B R C A 1$ and $B R C A 2$ result in a significant increase in cancer risk, these types of mutations represent only a small percentage of all tumours. ${ }^{67}$ The vast majority of cancers result from somatic alterations within cells resulting in a selective growth advantage over the well-controlled mechanisms of normal cell growth. ${ }^{68-71}$ There are currently technologies available for identifying and studying the molecular mechanisms defining various subtypes of common cancers and incorporating these data into the drug discovery and development process. The characterisation and understanding of genetic alterations driving tumourigenesis has already led to targeted oncology therapies with increased benefit to these molecularly

Table I. Selected examples of pharmacogenomics studies investigating currently available therapies.

\begin{tabular}{|c|c|c|c|c|}
\hline Therapeutic & Indication & Phenotype investigated & Gene/genomic relationship & References \\
\hline Carbamazepine & Seizures & Stevens-Johnson syndrome & HLA-B* 1502 & 56 \\
\hline Metoprolol & Hypertension & Blood pressure lowering & Beta-I adrenergic receptor & 57 \\
\hline \multirow[t]{3}{*}{ Abacavir } & HIV & Hypersensitivity & HLA-B*570I & 58,59 \\
\hline & & & HLA-DR7 & \\
\hline & & & HLA-DQ3 & \\
\hline Raloxifene & Osteoporosis & Bone mineral density & Vitamin D receptor & 60 \\
\hline Tolcapone & Parkinson's disease & Elevated liver transaminase levels & UDP-glucuronosyltransferase IA & 61 \\
\hline $\begin{array}{l}\text { Azathiopurine, } \\
\text { mercaptopurine, } \\
\text { thioguanine }\end{array}$ & Childhood leukaemia & Drug exposure & $\begin{array}{l}\text { Thiopurine S-methyltransferase- } \\
\text { inactivating mutation }\end{array}$ & 62 \\
\hline Zileuton & Asthma & FEVI response & 5-lipoxygenase promoter variant & 63 \\
\hline Fluvoxamine & Depression & $\begin{array}{l}\text { Drug response based on } \\
\text { Hamilton Depression Rating Scale }\end{array}$ & Serotonin transporter & 64 \\
\hline Sertraline & Depression & Time to therapeutic response & Serotonin transporter & 65 \\
\hline Enalaprilat & Hypertension & Blood pressure lowering & ACE Ins/Del & 66 \\
\hline
\end{tabular}

$\mathrm{ACE}=$ angiotensin-converting enzyme; $\mathrm{FEVI}=$ forced expiratory volume in one second. 
defined patient populations. The approval of Herceptin in 1998 paralleled the explosion of new scientific data emerging from the Human Genome Project. Scientists at Genentech recognised that a certain percentage of women with breast cancer overexpressed the Her-2 neu protein and that their cancer was particularly aggressive. ${ }^{72}$ In cells overexpressing the Her-2 neu protein it was discovered that targeted reduction in the expression of the protein returned the cancerous cells to a normal state, leading to the development of a monoclonal antibody which attaches to the Her-2 neu receptor to prevent the cell growth response. ${ }^{73,74}$ The overexpression of the Her-2 neu gene is observed in approximately 30 per cent of breast cancers, and it is this 30 per cent of patients that appreciate the optimal benefit from this targeted therapy. ${ }^{75}$ Based on these data, current Herceptin therapy requires women with advanced breast cancer to be screened for Her-2 neu overexpression through fluorescence in situ hybridisation or quantitative protein measures.

Gleevec, approved in 2001 for chronic myeloid leukaemia, treats a subset of patients who possess a genetic rearrangement known as the Philadelphia chromosome, in which a translocation of chromosome 9q34 to 22q11 [t(9;22) (q34;q11)] occurs, resulting in a breakpoint cluster region that fuses two genes, $B C R$ and the oncogene $A b l$, resulting in a constitutively active oncogenic product that plays an important role in cell growth. At least 90 per cent of patients with chronic myeloid leukaemia harbour this translocation, providing genetic evidence that it plays a role in disease risk. ${ }^{76}$ Once again, gaining a clear understanding of the molecular entity driving the disease, scientists at Novartis developed Gleevec, an inhibitor of BCR-ABL tyrosine kinase. A Phase II study reported in 2002 showed major cytogenetic responses in 60 per cent of subjects $(n=454)$ and complete haematological responses in 95 per cent of subjects who had previously failed on treatment with interferon-alpha. ${ }^{77}$

Genetic studies have also been instrumental in elucidating mechanisms of resistance to cancer therapy. Several reports have shown that the cause of Gleevec resistance in advanced phase disease is due to identified somatic point mutations within the ABL kinase domain in a subset of patients, which could possibly interfere with Gleevec binding. ${ }^{78-80}$

More recently, the elucidation of the response to the tyrosine kinase inhibitor Iressa has again highlighted the importance of characterising the somatic alterations contributing to tumourigenesis and the relationship between the mutations to increase the confidence in target mechanisms and drug response. ${ }^{69}$ In clinical development, some 10 per cent of patients with non-small cell lung cancer demonstrated a significant benefit with Iressa. When Lynch et al. examined the EGFR gene sequence - the target for Iressa action - in tumour DNA isolated from a subset of these responders, gainof-function mutations were identified in eight out of nine responders, compared with zero out of seven non-responders. ${ }^{81}$ Paez et al. examined the EGFR gene in DNA obtained from non-small cell lung cancer tumour samples and found similar mutations in one out of 61 tumour DNAs from USbased patients, while in Japanese patients the incidence rose to 15 out of 58 tumour DNAs, providing data correlating with an observed increase in response rates to Iressa in Japan. ${ }^{69}$

These data reinforce the importance of characterising underlying genetic heterogeneity within large tumour types. A priori knowledge of specific genetic markers indicative of drug response may influence the design of early proof-ofconcept clinical trials. In the case mentioned above, screening for those 10 per cent of subjects with EGFR mutations for enrolment would have actually increased the power of the development studies substantially by improving the efficacy rates through reducing intersubject variability in tumour heterogeneity. Studies have also shown the power of utilising expression-profiling patterns of selected signature genes in defining prognostic criteria, as exemplified by a recent report defining prognostic criteria for metastatic disease across multiple tumour types. ${ }^{82}$ The incorporation of these molecular signatures into a drug discovery and clinical development programme may influence many different phases, including the selection of the optimal target based upon the role it plays

Table 2. Current oncology products with a pharmacogenomics relationship.

\begin{tabular}{|c|c|c|c|c|c|}
\hline Therapy & Developer & Date approved & Primary indication & Genetic alteration & References \\
\hline Herceptin & Genentech & September 1998 & $\begin{array}{l}\text { Metastatic breast cancer } \\
\text { in tumours overexpressing } \\
\text { HER2 protein }\end{array}$ & $\begin{array}{l}\text { Amplification of the } \\
\text { Her-2 neu gene }\end{array}$ & $73-76$ \\
\hline Gleevec & Novartis & May 2001 & $\begin{array}{l}\text { Chronic myeloid } \\
\text { leukaemia }\end{array}$ & $\begin{array}{l}\text { Chromosomal translocation, } \\
\text { resulting in fusion of } B R C \\
\text { and the oncogene } A b l \text {, } \\
\text { resulting in activated } \\
\text { oncogene }\end{array}$ & $77-80$ \\
\hline Iressa & AstraZeneca & May 2003 & $\begin{array}{l}\text { Advanced or metastatic } \\
\text { non-small-cell lung cancer }\end{array}$ & $\begin{array}{l}\text { Activating mutations } \\
\text { in EGFR }\end{array}$ & 69,81 \\
\hline
\end{tabular}


in the molecular aetiology of the tumour subtype; the potential to allow enrichment of a patient population with molecularly defined tumours, as exemplified above and, finally, enrichment of the patient population most at risk for disease progression, to clearly test for drug effect.

\section{Conclusion}

As exemplified by the oncology examples highlighted in Table 2, pharmacogenomics will have an impact on optimal target selection and will increase efficacy in clinical development by targeting patient populations based on genetically defined disease phenotypes. It will also, ultimately, influence patient care when it provides a clear benefit to physicians' ability to deliver better medical care to patients. This will require a clear demonstration of benefit over standard assessments used in clinical practice, or when the molecular basis of the disease is so well understood that the clear path for development requires genomic knowledge to advance drug development. Advancements in genomic technologies, characterisation of human population genetic variation, enhancements in disease phenotyping methodologies, advances in informed consent processes and patient confidentiality technologies and the integration of molecular science with that of traditional clinical practice are paving the way for novel pharmacogenomic discoveries aimed at characterising disease risk, contributing to novel therapeutics and providing clues into the optimal treatment regimen based on a combination of clinically and genetically defined disease. While we are still in the early stages of this revolution, significant opportunities can be envisaged for scientific innovation to shape the future of medical care and patient health for many years to come.

\section{Acknowledgments}

Special thanks go to Paul Feeney, John Thompson, Duncan McHale and Michelle Penny for contributions to this manuscript. We especially thank our colleagues in the Pharmacogenomics groups for their passion and dedication in pursuing scientific research directed at improving human health.

\section{References}

1. Collins, F.S., Guyer, M.S. and Chakravarti, A. (1997), 'Variations on a theme: Cataloging human DNA sequence variation', Science Vol. 278, pp. $1580-1581$.

2. Chakravarti, A. (1998), 'It's raining SNPs, hallelujah?', Nat. Genet. Vol. 19, pp. 216-217.

3. International Human Genome Sequencing Consortium (2001), 'Initial sequencing and analysis of the human genome', Nature Vol. 409, pp. $860-921$.

4. Venter, J.C., Adams, M.D., Myers, E.W. et al. (2001), 'The sequence of the human genome', Science Vol. 291, pp. 1304-1351.

5. The International SNP Working Group (2001), 'A map of the human genome sequence variation containing 1.42 million single nucleotide polymorphisms', Nature Vol. 409, pp. 928-933.

6. The SNP Consortium (2002). http://snp.cshl.org/index.html. (Accessed 21st October, 2004).
7. The International HapMap Consortium (2003), 'The international HapMap project', Nature Vol. 426, pp. 789-796.

8. Wang, D.G., Fan, J., Siao, C. et al. (1998), 'Large scale identification, mapping and genotyping of single-nucleotide polymorphisms in the human genome', Science Vol. 280, pp. 1077-1082.

9. Hellio Le Graverand-Gastineau, M.P., Pickering, E., Chan, G. et al. (2003), 'Prevalence of radiographic osteoarthritis in subjects who are clinically asymptomatic', Abstract OARSI World Congress, 12-15th October.

10. Tamminen, M., Kakko, S., Kesaniemi, A. and Savolainen, M.J. (1996), 'A polymorphic site in the $3^{\prime}$ untranslated region of the cholesteryl ester transfer protein (CETP) gene is associated with low CETP activity', Atherosclerosis Vol. 124, pp. 237-247.

11. McCarthy, J.J., Parker, A., Salem, R. et al. for the GeneQuest Investigators (2004), 'Large scale association analysis for identification of genes underlying premature coronary heart disease: Cumulative perspective from analysis of 111 candidate genes', J. Med. Genet. Vol. 41, pp. 334-341.

12. Jurinke, C., van den Boom, D., Cantor, C.R. and Koster, H. (2002), 'The use of MassARRAY technology for high throughput genotyping', Methods Mol. Biol. Vol. 187, pp. 179-192.

13. Barker, D.L., Hansen, M., Faruqi, A.F. et al. (2004), 'Two methods of whole-genome amplification enable accurate genotyping across a 2320SNP linkage panel', Genome Res. Vol. 14, pp. 901-907.

14. Janne, P.A., Li, C., Zhao, X. et al. (2004), 'High-resolution singlenucleotide polymorphism array and clustering analysis of loss of heterozygosity in human lung cancer cell lines', Oncogene Vol. 23, pp. $2716-2726$.

15. Patil, N., Berno, A.J., Hinds, D.A. et al. (2001), 'Blocks of limited haplotype diversity revealed by high-resolution scanning of human chromosome 21', Science Vol. 294, pp. 1719-1723.

16. MacNeil, J.S. (2004), '(Still) looking for a few new sequencing technologies', Genome Technol. Vol. 46, pp. 23-26.

17. Robertson, J.A. (2001), 'Consent and privacy in pharmacogenetic testing', Nat. Genet. Vol. 28, pp. 207-209.

18. McIntyre, E.A. and Walter, M. (2002), 'Genetics of type 2 diabetes and insulin resistance: Knowledge from human studies', Clin. Endocrinol. Vol. 57, pp. 303-311.

19. Florez, J.C., Hirschhorn, J. and Altshuler, D. (2003), 'The inherited basis of diabetes mellitus: Implications for the genetic analysis of complex traits', Annu. Rev. Genomics Hum. Genet. Vol. 4, pp. 257-291.

20. Allison, D.B., Faith, M.S. and Nathan, J.S. (1996), 'Risch's lambda values for human obesity', Int. J. Obes. Vol. 20, pp. 990-999.

21. Maes, H.H., Neale, M.C. and Eaves, L.J. (1997), 'Genetic and environmental factors in relative body weight and human adiposity', Behav. Genet. Vol. 27, pp. 325-351.

22. Lanyon, P., Muir, K., Doherty, S. and Doherty, M. (2000), 'Assessment of a genetic contribution to osteoarthritis of the hip: Sibling study', BMJ Vol. 321, pp. 1179-1183.

23. MacGregor, A.J., Antoniades, L., Matson, M. et al. (2000), 'The genetic contribution to radiographic hip osteoarthritis in women', Arthritis Rheum. Vol. 43, pp. 2410-2416.

24. Inazu, A., Brown, M.L., Hesler, C.B. et al. (1990), 'Increased high-density lipoprotein levels caused by a common cholesteryl-ester transfer protein gene mutation', N. Engl. J. Med. Vol. 323, pp. 1234-1238.

25. Yamashita, S., Sprecher, D.L., Sakai, N. et al. (1990), 'Accumulation of apolipoprotein E-rich high density lipoproteins in hyperalphalipoproteinemic human subjects with plasma cholesteryl ester transfer protein deficiency', J. Clin. Invest. Vol. 86, pp. 688-695.

26. Macchi, P., Villa, A., Giliani, S. et al. (1995), 'Mutations of Jak-3 gene in patients with autosomal severe combined immune deficiency (SCID)', Nature Vol. 377, pp. 65-68.

27. Clark, R.W., Sutfin, T.A., Ruggeri, R.B. et al. (2004), 'Raising highdensity lipoprotein in humans through inhibition of cholesteryl ester transfer protein: An initial multidose study of torcetrapib', Arterioscler. Thromb. Vasc. Biol. Vol. 24, pp. 490-497.

28. Changelian, P.S., Flanagan, M.E., Ball, D.J. et al. (2003), 'Prevention of organ allograft rejection by a specific Janus kinase 3 inhibitor', Science Vol. 30, pp. $875-881$. 
29. Steffansson, H., Sarginson, J., Kong, A. et al. (2002), 'Neuregulin I and susceptibility to schizophrenia', Am. J. Hum. Genet. Vol. 71, pp. 877-892.

30. Gretarsdottir, S., Thorleifsson, G., Reynisdottir, S.T. et al. (2003), 'The gene encoding phosphodiesterase 4D confers risk of ischemic stroke', Nat. Genet. Vol. 35, pp. 131-138.

31. Dwyer, J.H., Allayee, H., Dwyer, K.M. et al. (2004), 'Arachidonate 5 -lipoxygenase promoter genotype, dietary arachidonic acid, and atherosclerosis', N. Engl. J. Med. Vol. 350, pp. 29-37.

32. Mank-Seymour, A.R., Durham, L.K., Thompson, J.F. et al. (2004), 'Association between single-nucleotide polymorphisms in the endothelial lipase (LIPG) gene and high-density lipoprotein cholesterol levels', Biochim. Biophys. Acta Vol. 1636, pp. 40-46.

33. Devlin, B. and Risch, N. (1995), 'A comparison of linkage disequilibrium measures for fine-scale mapping', Genomics Vol. 29, pp. 311-322.

34. Carlson, C.S., Eberle, M.A., Rieder, M.J. et al. (2004), 'Selecting a maximally informative set of single-nucleotide polymorphisms for association analyses using linkage disequilibrium', Am. J. Hum. Genet. Vol. 74, pp. 106-120.

35. Ogura, Y., Bonen, D.K., Inohara, N. et al. (2004), 'Association of NOD2 leucine-rich repeat variants with susceptibility to Crohn's disease', Nature Vol. 411, pp. 603-606.

36. Hinds, D.A., Seymour, A.B., Durham, L.K. et al. (2004), 'Application of pooled genotyping to scan candidate regions for association with HDL levels', Hum. Genomics Vol. 1, pp. 421-434.

37. Stephens, J.C., Schneider, J.A., Tanguay, D.A. et al. (2001), 'Haplotype variation and linkage disequilibrium in 313 human genes', Science Vol. 293, pp. $489-493$.

38. Thompson, J.F., Lira, M.E., Durham, L.K. et al. (2003), 'Polymorphisms in the CETP gene and association with CETP mass and HDL levels', Atherosclerosis Vol. 167, pp. 195-204.

39. Sachse, C., Brockmoller, J., Bauer, S. and Roots, I. (1997), 'Cytochrome P450 2D6 variants in a Caucasian population: Allele frequencies and phenotypic consequences', Am. J. Hum. Genet. Vol. 60, pp. 284-295.

40. McElroy, S.M., Sachse, C., Richmond, J. et al. (2000), 'Utilization of CYP450 2D6 genotyping as an alternative to probe drug phenotyping for determination of metabolic status in a clinical setting', AAPS Pharmsci. Vol. 2(33), available at http://www.aapsharmsci.org/view. asp?art $=$ ps020433. (Accessed 21st October, 2004).

41. Evans, W.E. and Relling, M.V. (1999), 'Pharmacogenomics: Translating functional genomics into rational therapeutics', Science Vol. 286, pp. $487-491$.

42. Wolf, C.R. and Smith, G. (1999), 'Pharmacogenetics', Br. Med. Bull. Vol. 55, pp. 366-386

43. Ingelman-Sundberg, M., Oscarson, M. and McLellan, R.A. (1999), 'Polymorphic human cytochrome P450 enzymes: An opportunity for individualized drug treatment', Trends Pharmacol. Sci. Vol. 20, pp. 342-349.

44. Ingelman-Sundberg, M. (2001), 'Pharmacogenetics: An opportunity for a safer and more efficient pharmacotherapy', J. Intern. Med. Vol. 250, pp. $186-200$.

45. Goldstein, J.A. (2001), 'Clinical relevance of genetic polymorphisms in the human CYP 2C subfamily', Br. J. Clin. Pharmacol. Vol. 52, pp. 349-355.

46. Ozdemir, V., Tyndale, R.F., Reed, K. et al. (1999), 'Paroxetine steady-state plasma concentration in relation to CYP2D6 genotype in extensive metabolizers', J. Clin. Psychopharmacol. Vol. 19, pp. 472-475.

47. Charlier, C., Broly, F., Lhermitte, M. et al. (2003), 'Polymorphisms in the CYP 2D6 gene: Association with plasma concentrations of fluoxetine and paroxetine', Ther. Drug Monit. Vol. 25, pp. 738-742.

48. Helgadottir, A., Manolescu, A., Thorleifsson, G. et al. (2004), 'The gene encoding 5-lipoxygenase activating protein confers risk of myocardial infarction and stroke', Nature Genet. Vol. 36, pp. 233-239.

49. Dean, M., Carrington, M., Winkter, C. et al. (1996), 'Genetic restriction of HIV-1 infection and progression to AIDS by a deletion allele of the CKR5 structural gene', Science Vol. 273, pp. 1856-1861.

50. Penny, M., Myrand, S., Lin, C. et al. (2004), 'Pharmacogenetic analysis of polymorphisms in the chemokine receptors CCR 5 and CCR 2 in the clinical development of a CCR 5 antagonist (UK-427, 857) for the treatment of HIV/AIDS', FDA Science Forum, Washington, DC, USA.

51. Lesko, L.J., Salerno, R.A., Spear, B.B. et al. (2003), 'Pharmacogenetics and pharmacogenomics in drug development and regulatory decision-making:
Report of the first FDA-PWG-PhRMA-DruSafe workshop', J. Clin. Pharmacol. Vol. 43, pp. 342-358.

52. Roses, A.D. (2002), 'Genome-based pharmacogenetics and the pharmaceutical industry', Nat. Rev. Genet. Vol. 1, pp. 541-549.

53. Danoff, T.M., Campbell, D.A., McCarthy, L.C. et al. (2004), 'A Gilbert's syndrome UGT1A1 variant confers susceptibility to Tranilast-induced hyperbilirubinemia', Pharmacogenomics J. Vol. 4, pp. 49-53.

54. Roses, A.D. (2004), 'Pharmacogenetics and drug development: The path to safer and more effective drugs', Nat. Rev. Genet. Vol. 5, pp. 645-655.

55. Xu, C.F., Lewis, K.F., Yeo, A.J. et al. (2004), 'Identification of a pharmacogenetic effect by linkage disequilibrium mapping', Pharmacogenomics $J$. (in press).

56. Chung, W.-H., Hung, S.-I., Hong, H.-S. et al. (2004), 'A marker for Stevens-Johnson syndrome', Nature Vol. 428, p. 486

57. Johnson, J.A., Zineh, I., Puckett, B.J. et al. (2003), 'Beta-1 adrenergic receptor polymorphisms and antihypertensive response to metoprolol', Clin. Pharmacol. Ther. Vol. 74, pp. 44-52

58. Mallal, S., Nolan, D., Witt, C. et al. (2002), 'Association between presence of HLA-B*5701, HLA-DR7, and HLA-DQ3 and hypersensitivity to HIV-1 reverse-transcriptase inhibitor abacavir', Lancet Vol. 359, pp. 727-732.

59. Hetherington, S., Hughes, A.R., Mosteller, M. et al. (2002), 'Genetic variations in HLA-B region and hypersensitivity reactions to abacavir', Lancet Vol. 359, pp. 1121-1122.

60. Palumbo, S., Giuliano, F., Mossetti, G. et al. (2003), 'Raloxifene administration in post-menopausal women with osteoporosis: Effect of different BsmI vitamin D receptor genotypes', Hum. Reprod. Vol. 18, pp. $192-198$.

61. Acuna, G., Foernzler, D., Leong, D. et al. (2002), 'Pharmacogenetic analysis of adverse drug effect reveals genetic variant for susceptibility to liver toxicity', Pharmacogenomics J. Vol. 2, pp. 327-334.

62. Krynetski, E.Y. and Evans, W.E. (2000), 'Genetic polymorphism of thiopurine S-methyltransferase: Molecular mechanisms and clinical importance', Pharmacology Vol. 61, pp. 136-146.

63. Drazen, J.M., Yandava, C.N., Dube, L. et al. (1999), 'Pharmacogenetic association between ALOX5 promoter genotype and the response to antiasthma treatment', Nat. Genet. Vol. 22, pp. 168-170.

64. Smeraldi, E., Zanardi, R., Benedetti, F. et al. (1998), 'Polymorphism within the promoter of the serotonin transporter gene and antidepressant efficacy of fluvoxamine', Mol. Psychiatry Vol. 3, pp. 508-511.

65. Durham, L.K., Webb, S.M., Milos, P.M. et al. (2004), 'The serotonin transporter polymorphism, 5HTTLPR, is associated with a faster response time to sertraline in an elderly population with major depression', Psychoparmacol. Vol. 174, pp. 525-529.

66. Ueda, S., Meredith, P.A., Morton, J.J. et al. (1998), 'ACE (I/D) genotype as a predictor or the magnitude and duration of the response to an ACE inhibitor drug (enalaprilat) in humans', Circulation Vol. 98, pp. $2148-2153$.

67. Iau, P.T., Macmillan, R.D. and Blamey, R.W. (2001), 'Germline mutations associated with breast cancer susceptibility', Eur. J. Cancer Vol. 37, pp. $300-321$.

68. Wang, Z., Shen, D., Parsons, D.W. et al. (2004), 'Mutational analysis of the tyrosine phosphatome in colorectal cancers', Science Vol. 304, pp. 1164-1166.

69. Paez, J.G., Psai, A. and Janne, P.A. (2004), 'EGFR mutations in lung cancer: Correlation with clinical response to gefitinib therapy', Science Vol. 304, pp. 1497-1500.

70. Bardelli, A., Parsons, D.W., Silliman, N. et al. (2003), 'Mutational analysis of the tyrosine kinome in colorectal cancers', Science Vol. 300, p. 949.

71. Pollock, P.M., Harper, U.L., Hansen, K.S. et al. (2002), 'High frequency of BRAF mutations in nevi', Nat. Genet. Vol. 33, pp. 19-20.

72. Slamon, D.J., Clark, G.M., Wong, S.G. et al. (1987), 'Human breast cancer: Correlation of relapse and survival with amplification of the HER-2/neu oncogene', Science Vol. 235, pp. 177-182.

73. Shepard, H.M., Lewis, G.D., Sarup, J.C. et al. (1991), 'Monoclonal antibody therapy of human cancer: Taking the HER 2 protooncogene to the clinic', J. Clin. Immunol. Vol. 11, pp. 117-127.

74. Molina, M.A., Codony-Servat, J., Albanell, J. et al. (2001), 'Trastuzumab (herceptin), a humanized antiHer2 receptor monoclonal antibody, inhibits 
basal and activated Her2 ectodomain cleavage in breast cancer cells', Cancer Res. Vol. 61, pp. 4744-4749.

75. Cobleigh, M.A., Vogel, C.L., Tripathy, D. et al. (1999), 'Multinational study of the efficacy and safety of humanized anti-HER 2 monoclonal antibody in women who have HER2-overexpressing metastatic breast cancer that has progressed after chemotherapy for metastatic disease', J. Clin. Oncol. Vol. 17, pp. 2639-2648.

76. Rowley, J.D. (1973), 'A new consistent chromosomal abnormality in chronic myelogenous leukaemia identified by quinacrine fluorescence and Giesma staining', Nature Vol. 243, pp. 290-293.

77. Kantarjian, H., Sawyers, C., Hochhaus, A. et al. (2002), 'Hematologic and cytogenetic responses to imatinib mesylate in chronic myelogenous leukaemia', N. Engl. J. Med. Vol. 346, pp. 645-652.

78. Gorre, M.E., Mohammed, M., Ellwood, K. et al. (2001), 'Clinical resistance to STI-571 cancer therapy caused by BCR-ABL gene mutation or amplification', Science Vol. 293, pp. 876-880.
79. Bubnoff, N.V., Schneller, F., Peschel, C. and Duyster, J. (2002), 'BCR$A B L$ gene mutations in relation to clinical resistance of Philadelphiachromosome-positive leukaemia to STI571: A prospective study', Lancet Vol. 359, pp. 487-491.

80. Branford, S., Rudzki, Z., Walsh, S. et al. (2002), 'High frequency of point mutations clustered within the adenosine triphosphate-binding region of $\mathrm{BCR} / \mathrm{ABL}$ in patients with chronic myeloid leukemia or $\mathrm{Ph}$-positive acute lymphoblastic leukemia who develop imatinib (STI571) resistance', Blood Vol. 99, pp. 3472-3475.

81. Lynch, T.J., Bell, D.W., Sordella, R. et al. (2004), 'Activating mutations in the epidermal growth factor receptor underlying responsiveness of non-small-cell lung cancer to gefitinib', N. Engl. J. Med. Vol. 350, pp. $350-361$.

82. Ramaswamy, S., Ross, K.N., Lander, E.S. and Golub, T.R. (2003), 'A molecular signature of metastasis in primary solid', Nat. Genet. Vol. 33, pp. $49-54$. 